\title{
Almost Sure Convergence to Consensus in Markovian Random Graphs
}

\author{
Ion Matei, Nuno Martins and John S. Baras
}

\begin{abstract}
In this paper we discuss the consensus problem for a network of dynamic agents with undirected information flow and random switching topologies. The switching is determined by a Markov chain, each topology corresponding to a state of the Markov chain. We show that in order to achieve consensus almost surely and from any initial state the sets of graphs corresponding to the closed positive recurrent sets of the Markov chain must be jointly connected. The analysis relies on tools from matrix theory, Markovian jump linear systems theory and random processes theory. The distinctive feature of this work is addressing the consensus problem with "Markovian switching" topologies.
\end{abstract}

\section{INTRODUCTION}

A consensus problem, which lies at the foundation of distributed computing, consists of a group of dynamic agents who seek to agree upon certain quantities of interest by exchanging information among them according to a set of rules. This problem can model many phenomena involving information exchange between agents such as cooperative control of vehicles, formation control, flocking, synchronization, parallel computing, etc. Thus the consensus problem has been widely studied in the literature. Distributed computation over networks has a long history in control theory starting with the work of Borkar and Varaiya [1], Tsitsikils, Bertsekas and Athans [15], [16], on asynchronous agreement problems and parallel computing. Olfati-Saber and Murray introduced in [11], [12] the theoretical framework for solving consensus problems. Jadbabaie et al. studied in [6] alignment problems involving reaching an agreement. Relevant extensions of the consensus problem were done by Ren and Beard [10] and by Moreau in [8].

The communication networks between agents may change in time due to link failures, packet drops, appearance or disappearance of nodes etc. Many of the variations in topology may happen randomly which lead to considering consensus problems under a stochastic framework. Hatano and Mesbahi consider in [7] an agreement problem over random information networks where the existence of an information channel between a pair of elements at each time instance is probabilistic and independent of other channels. In [14] Salehi and Jadbabaie provide necessary and sufficient conditions for reaching consensus in the case of a discrete

Ion Matei, Nuno Martins and John S. Baras are with the Institute for Systems Research and the Department of Electrical and Computer Engineering, University of Maryland, College Park, imatei, nmartins, baras @ umd. edu

The research of Ion Matei and John Baras was supported by NASA under Cooperative Agreements NCC8-235 and NAG3- 2844, and by the U.S. ARL under the CTA Program, Communications and Networks Consortium, Cooperative Agreement DAAD19-01-2-0011. The research of Nuno Martins was supported by the NSF under NSF EECS 0644764 CAREER Award. linear system, where the communication flow is given by a random graph process, independent of other time instances. Under a similar model of the communication topology, Porfiri and Stilwell give sufficient conditions for almost sure convergence to consensus in [9].

In this paper we consider the discrete-time consensus problem for a group of dynamic agents with undirected information flow and random switching topologies. The switching process is governed by a Markov chain whose states correspond to possible communication topologies. The advantage of having Markovian switching topologies resides in their ability to model a topology change which depends on the previous communication topology. Consider the example of an agent which may adjust the power allocated to transmissions in order to overcome the failure of a link due to a large distance between agents. The actions of such an agent determines a change in the communication topology which is dependent on the state of the network at previous time instants. Our model is more general since it also includes the case of independent random changes in the communication flow; a case which benefited from significant attention in the literature.

Notations: We will denote by $\mathbb{1}$ the $n$-dimensional vector of all ones. We will use the same symbol for a vector of all ones with $n^{2}$ entries. It will be clear from the context what dimension vector $\mathbb{1}$ has. Let $\left\{F_{i}\right\}_{i=1}^{s}$ be a set of matrices. By $\operatorname{diag}\left(\left\{F_{i}\right\}_{i=1}^{s}\right)$ we understand a block diagonal matrix having on the block diagonal matrices $F_{1}, \ldots, F_{s}$. The matrix $\operatorname{diag}\left(\frac{1}{n} \mathbb{1}^{T}\right)$ is a block diagonal matrix having on the block diagonal $n \times n$ matrices of all ones; $\operatorname{diag}\left(\frac{1}{n^{2}} \mathbb{1} \mathbb{1}^{T}\right)$ represents a block diagonal matrix with $n^{2} \times n^{2}$ matrices of all ones on the main block diagonal. The symbol $\otimes$ denotes the Kronecker product.

The outline of the paper is as follows. In Section II we present the setup and formulation of the problem. In Section III we state our main result and give an intuitive explanation. In Section IV we provide first a set of theoretical tools used in proving the main result and then we proceed with the proof of the main theorem. In Section V we briefly discuss extensions of our result.

\section{Problem FORMULATION}

In this section we introduce the problem setup for the almost sure convergence to consensus within a discrete-time context. We consider a group of $n$ dynamic agents for which the information flow is modeled as an undirected graph $G=$ $(\mathcal{V}, \mathcal{E}, A)$ of order $n$. The set $\mathcal{V}=\{1, \ldots, n\}$ represents the set of vertices, $\mathcal{E} \subseteq \mathcal{V} \times \mathcal{V}$ is the set of edges and $A=\left[a_{i j}\right]$ is a symmetric adjacency matrix with $a_{i j}$ being positive if 
a link exists between vertices $(i, j)$ and zero otherwise. By graph Laplacian we understand the matrix $L$ whose entries are:

$$
l_{i j}= \begin{cases}\sum_{k=1, k \neq i}^{n} a_{i k} & j=i \\ -a_{i j} & j \neq i\end{cases}
$$

with $i \in\{1, \ldots, n\}$. Equivalently we can write the Laplacian of an undirected graph as $L=D-A$, where $A$ is the adjacency matrix of the graph and $D$ is the diagonal matrix of vertex degrees $d_{i i}=\sum_{i \neq j} a_{i j}$. Throughout this paper we will consider (except Section V) undirected graphs.

Let $s$ be a positive integer and let $\theta(k)$ be a finite-state homogeneous Markov chain which takes values in a discrete set $\mathcal{S}=\{1, \cdots, s\}$, with $P$ its $s \times s$ transition probability matrix, $P=\left(p_{i j}\right)$ (rows sum up to one).

Definition 2.1: (Discrete-Time Markovian Random Graph) Given a positive integer $s$, let $\mathcal{G}=\left\{G_{i}\right\}_{i=1}^{s}$ be a set of (undirected) graphs of order $n$. By a discrete-time Markovian random graph (DTMRG) we understand a map G from $\mathcal{S}$ to $\mathcal{G}$, such that

$$
\mathbf{G}(\theta(k))=G_{\theta(k)}
$$

for all positive integer values of $k$. In what follows this map will be directly referred by $G_{\theta(k)}$. We note the $G_{\theta(k)}$ is a discrete finite-state Markovian process, whose probabilistic description is given by the probability transition matrix $P$.

We denote by $X(k)$ the $n$-dimensional column vector representing the state of the agents. We assume that the information flow among agents is described by a DTMRG $G_{\theta(k)}$ and we consider a linear discrete stochastic dynamic system governing the evolution of the state vector:

$$
X(k+1)=F_{\theta(k)} X(k), X(0)=X_{0},
$$

where the $n \times n$ random matrix $F_{\theta(k)}$ represents the state's updating rule corresponding to the current communication graph and it takes values in a finite set of matrices $\left\{F_{i}\right\}_{i=1}^{s}$. The initial condition $X_{0}$ is considered deterministic.

We define the agreement space as the subspace generated by the vector of all ones $\mathcal{A}=\operatorname{span}(\mathbb{1})$, where we denote by $\mathbb{1}$ the $n$-dimensional vector of all ones.

Definition 2.2: We say that the vector $X(k)$ converges almost surely to consensus if it asymptotically reaches the agreement space in the almost sure sense

$$
X(k) \stackrel{a . s}{\longrightarrow} \mathcal{A} \text {. }
$$

Definition 2.3: We say that the state vector $X(k)$ reaches average consensus almost surely if

$$
X(k) \stackrel{a . s}{\longrightarrow} a v\left(X_{0}\right) \mathbb{1} .
$$

Problem 2.1: Given a DTMRG $G_{\theta(k)}$ and the state's updating rule $F_{\theta(k)}$, we derive necessary and sufficient conditions such that the state vector $X(k)$, evolving according to (1), converges almost surely to average consensus for any initial state $X_{0}$.
For the most part of this paper we will consider an updating rule given by

$$
F_{i}=I-\epsilon L_{i}, i \in \mathcal{S},
$$

where $L_{i}$ is the Laplacian of the undirected graph $G_{i}$ and $\epsilon<1 / \max _{i}\left\{\sum_{j \neq i} l_{i j}\right\}$. Let this updating rule be referred to as protocol A1. Note that protocol A1 is a nearest neighbor updating rule and it is appealing for its distributed implementability.

\section{MAIN RESUlT}

In this section we introduce the necessary and sufficient conditions for reaching average consensus in the almost sure sense together with some intuitive explanations of these conditions. We defer the rigorous mathematical proof for Section IV.

Let us first introduce the following definition borrowed from [6].

Definition 3.1: (Jointly Connected Graphs) Given a positive integer $s$, let $\left\{G_{i}\right\}_{i=1}^{s}$ be a set of undirected graphs. We say that this set is jointly connected if the union of the graphs in the set generates a connected graph where by union we understand the union of the edges of all graphs in the set.

Consider the problem setup presented in Section II. By the Decomposition Theorem of the states of a Markov chain (see [5]) the state space $\mathcal{S}$ can be partitioned uniquely as

$$
\mathcal{S}=\left\{T \cup C_{1} \cup \cdots \cup C_{q}\right\},
$$

where $T$ is the set of transient states and $C_{1}, \ldots, C_{q}$ are irreducible closed sets of (positive) recurrent states. Since $\theta(k)$ is a finite state Markov chain there exists at least one (positive) recurrent closed set. We make the assumption that the distribution of $\theta(0)$ is such that the probability of $\theta(0)$ to belong to any of the sets $T$ or $C_{i}$ is non-zero. Let $\mathcal{G}_{i}=$ $\left\{G_{j_{1}}, G_{j_{2}}, G_{j_{\left|C_{i}\right|}}\right\}$ be the sets of graphs corresponding to the states in the sets $C_{i}$ with $i \in\{1, \ldots, q\}$ and where by $\left|C_{i}\right|$ we denote the cardinality of $C_{i}$.

Theorem 3.1: (almost sure convergence to average consensus) Consider the stochastic system (1). Then, under protocol $\mathrm{A} 1$, the random vector $X(k)$ converges almost surely to average consensus for any initial state $X_{0}$ if and only if each of the sets of graphs $\mathcal{G}_{i}$ corresponding to the closed sets $C_{i}$ are jointly connected.

We defer for the next section the proof of this theorem and rather provide here an intuitive explanation. Regardless of the initial state of $\theta(k)$, there exist a time instant after which $\theta(k)$ will be constrained to take values only in one of the closed sets $C_{i}$. Since $C_{i}$ are irreducible and (positive) recurrent the probability of $\theta(k)$ to visit each of the states belonging to $C_{i}$ will never converge to zero. Thus $\theta(k)$ will visit each of these states infinitely many times and consequently since the graphs corresponding to these states are jointly connected the agents will be connected infinitely many times. This is sufficient for the state vector $X(k)$ to converge to consensus. On the other hand if we assume the existence of at least one 
set $C_{i}$ such that the corresponding set of graphs $\mathcal{G}_{i}$ is not jointly connected, then with non-zero probability $\theta(k)$ may be isolated in such a set after a while. Since the graphs are not jointly connected, there will be at least one agent which will not exchange information with the others. This is enough to conclude (in the case of undirected graphs) that there exists initial states such that with non-zero probability, average consensus is not reached.

\section{Proof of the Main Result}

In this section we introduce a number of supporting results and their proofs and detail the proof of Theorem 3.1. We start by stating a number of results from the literature which will be useful in our analysis and then we continue with a series of corollaries and lemmas that will prove instrumental for showing Theorem 3.1.

Theorem 4.1: ([17]) Let $s$ be a positive integer and $\left\{A_{i}\right\}_{i=1}^{s}$ a finite set of $n \times n$ ergodic matrices with the property that for each sequence of matrices $A_{i_{1}}, \ldots, A_{i_{j}}$ of positive length the product matrix $A_{i_{j}} \cdots A_{i_{1}}$ is an ergodic matrix. Then for each infinite sequence $A_{i_{1}}, A_{i_{2}}, \ldots$ there exist a vector $c$ such that

$$
\lim _{j \rightarrow \infty} A_{i_{j}} A_{i_{j-1}} \cdots A_{i_{1}}=\mathbb{1} c^{T}
$$

Lemma 4.1: (Lemma 2, [6]) Let $m$ be a positive integer with $m \leq 2$ and let $\left\{A_{i}\right\}_{i=1}^{m}$ be a set of nonnegative $n \times n$ matrices. Suppose the diagonal entries of each matrix $A_{i}$ are all positive and let $\mu$ and $\rho$ denote the smallest and the largest of these respectively. Then the following property holds:

$$
A_{1} A_{2} \cdots A_{m} \geq\left(\frac{\mu^{2}}{2 \rho}\right)^{m-1}\left(A_{1}+A_{2}+\ldots+A_{m}\right)
$$

In the special case of doubly stochastic matrices we can derive the following corollary from Theorem 4.1.

Corollary 4.1: Under the assumptions of Theorem 4.1, if $A_{1}, \ldots, A_{s}$ are doubly stochastic, then for each infinite sequence $A_{i_{1}}, A_{i_{2}}, \ldots$ we have

$$
\lim _{j \rightarrow \infty} A_{i_{j}} A_{i_{j-1}} \cdots A_{i_{1}}=\frac{1}{n} \mathbb{1} \mathbb{1}^{T} .
$$

Proof: Follows immediately from Theorem 4.1 and from the doubly stochastic property of matrices $\left\{A_{i}\right\}_{i=1}^{s}$.

Lemma 4.2: Given a positive integer $s$, consider a set of jointly connected undirected graphs $\left\{G_{i}\right\}_{i=1}^{s}$ and a finite set of indices $\left\{i_{k}\right\}_{k=1}^{j}$ which contains (at least once) each of the values $\{1, \ldots, s\}$. Let $\left\{F_{i}\right\}_{i=1}^{s}$ be a set of $n \times n$ (symmetric) matrices given by $F_{i}=I-\epsilon L_{i}$ (as in (2)), where $L_{i}$ is the Laplacian of the graph $G_{i}$. Then the matrix product $F_{i_{1}} F_{i_{2}} \ldots F_{i_{j}}$ is ergodic. The same property holds for the matrix product $\left(F_{i_{1}} \otimes F_{i_{1}}\right)\left(F_{i_{2}} \otimes F_{i_{2}}\right) \ldots\left(F_{i_{j}} \otimes F_{i_{j}}\right)$.

Proof: The proof is based on Lemma 4.1 and it is similar to the proof of Lemma 1 in [6]. By construction all matrices $\left\{F_{i}\right\}_{i=1}^{s}$ are non-negative matrices with positive diagonal entries. Then by Lemma 4.1 we have

$$
F_{i_{1}} F_{i_{2}} \ldots F_{i_{j}} \geq \gamma\left(F_{1}+F_{2}+\ldots F_{s}\right),
$$

where $\gamma>0$ depends on the largest and smallest entry of matrices $\left\{F_{i}\right\}_{i=1}^{s}$. We can further write

$$
F_{i_{1}} F_{i_{2}} \ldots F_{i_{j}} \geq \gamma\left(F_{1}+F_{2}+\ldots F_{s}\right)=\gamma s F,
$$

where $F=I-\epsilon / s \sum_{i=1}^{s} L_{i}$. From the hypothesis that the set $\left\{G_{i}\right\}_{i=1}^{s}$ are jointly connected $L=\sum_{i=1}^{s} L_{i}$ corresponds to the Laplacian of a connected undirected weighted graph. Thus $F$ is a stochastic ergodic matrix and as a consequence there exist a finite positive number $k$ such that all entries of $F^{k}$ are positive. Then $\left(F_{i_{1}} F_{i_{2}} \ldots F_{i_{j}}\right)^{k} \geq \gamma^{k} F^{k}$ and therefore $\left(F_{i_{1}} F_{i_{2}} \ldots F_{i_{j}}\right)^{k}$ has all entries strictly positive which is enough to conclude its ergodicity. To derive the ergodicity of the matrix product involving the Kronecker product of matrices $F_{i}$ 's notice that $\left(\left(F_{i_{1}} \otimes F_{i_{1}}\right)\left(F_{i_{2}} \otimes\right.\right.$ $\left.\left.F_{i_{2}}\right) \ldots\left(F_{i_{j}} \otimes F_{i_{j}}\right)\right)^{k}=\left(F_{i_{1}} F_{i_{2}} \ldots F_{i_{j}}\right)^{k} \otimes\left(F_{i_{1}} F_{i_{2}} \ldots F_{i_{j}}\right)^{k}$ and since $\left(F_{i_{1}} F_{i_{2}} \ldots F_{i_{j}}\right)^{k}$ has all entries strictly positive the same is true for $\left(F_{i_{1}} F_{i_{2}} \ldots F_{i_{j}}\right)^{k} \otimes\left(F_{i_{1}} F_{i_{2}} \ldots F_{i_{j}}\right)^{k}$.

Lemma 4.3: Let $s$ be a positive integer and let $\left\{A_{i j}\right\}_{i, j=1}^{s}$ be a set of $n \times n$ doubly stochastic ergodic matrices. Let $P=\left(p_{i j}\right)$ be an $s \times s$ stochastic matrix corresponding to an irreducible (positive) recurrent Markov chain. Consider the $n s \times n s$ dimensional matrix $Q$ whose $(i, j)^{t h}$ block is defined as $Q_{i j}=p_{i j} A_{i j}$. Then

$$
\lim _{k \rightarrow \infty} Q^{k}=(\Phi \otimes I) \operatorname{diag}\left(\frac{1}{n} \mathbb{1} \mathbb{1}^{T}\right)
$$

where $\operatorname{diag}\left(\frac{1}{n} \mathbb{1}^{T}\right)$ is an $n s \times n s$ block diagonal with matrices $\frac{1}{n} \mathbb{1} \mathbb{1}^{T}$ on the main diagonal. The matrix $\Phi$ is a stochastic matrix whose entries depend on the properties of matrix $P$ :

$$
(\Phi)_{i j}= \begin{cases}\lim _{k \rightarrow \infty}\left(P^{k}\right)_{i j} & \text { if } P \text { is aperiodic } \\ \left(P^{l}\right)_{i j} & \text { if } P^{d}=P, l \in\{0, \ldots, d\}\end{cases}
$$

Proof: The proof of this lemma is based on Corollary 4.1. We can express the $(i, j)^{t h}$ block entry of matrix $Q^{k}$ as follows:

$$
\left(Q^{k}\right)_{i j}=\sum_{1 \leq i_{1}, \ldots i_{k-1} \leq n} p_{i i_{1}} A_{i i_{1}} p_{i_{1} i_{2}} A_{i_{1} i_{2}} \ldots p_{i_{k-1} j} A_{i_{k-1} j}
$$

Notice from (8) that as $k$ goes to infinity in each of the $(i, j)$ block of matrix $Q^{k}$ there will be infinite sums containing infinite products of ergodic (doubly stochastic) matrices of the form $A_{i i_{1}} A_{i_{1} i_{2}} \ldots$ By Corollary 4.1 every such infinite product of matrices converges to $\frac{1}{n} \mathbb{1} \mathbb{1}^{T}$. Therefore as $k$ goes to infinity we can express (8) as:

$$
\lim _{k \rightarrow \infty}\left(Q^{k}\right)_{i j}=\frac{1}{n} \mathbb{1}^{T} \lim _{k \rightarrow \infty} \sum_{1 \leq i_{1}, \ldots i_{k-1} \leq n} p_{i i_{1}} p_{i_{1} i_{2}} \ldots p_{i_{k-1} j}
$$

It is not difficult to observe that the right-hand sum in (9) is the $(i, j)^{t h}$ entry of the matrix $P^{k}$. Whether or not this 
sum will converge depends entirely on the properties of the Markov chain behind the probability transition matrix $P$. Thus as $k$ goes to infinity the matrix $\Phi=P^{k}$ will be a stochastic matrix as in (7).

Lemma 4.4: Let $s$ be a positive integer and consider a set of $n \times n$ matrices $\left\{F_{i}\right\}_{i=1}^{s}$ given by $F_{i}=I-\epsilon L_{i}$ (as in (2)) where $\left\{L_{i}\right\}_{i=1}^{s}$ are the Laplacians of $\left\{G_{i}\right\}_{i=1}^{s}$, a jointly connected set of undirected graphs of order $n$. Let $P$ be an $s \times s$ probability transition matrix corresponding to an irreducible (positive) recurrent finite-state Markov chain. Let $Q$ and $\tilde{Q}$ be defined as:

$$
Q=\operatorname{diag}\left(\left\{F_{i}\right\}_{i=1}^{s}\right)(P \otimes I)
$$

and

$$
\tilde{Q}=\operatorname{diag}\left(\left\{F_{i} \otimes F_{i}\right\}_{i=1}^{s}\right)(P \otimes I),
$$

where the matrices $\operatorname{diag}\left(\left\{F_{i}\right\}_{i=1}^{s}\right)$ and $\operatorname{diag}\left(\left\{F_{i} \otimes F_{i}\right\}_{i=1}^{s}\right)$ are $n s \times n s$ and $n^{2} s \times n^{2} s$ respectively block diagonal matrices. Then

$$
\begin{aligned}
& \lim _{k \rightarrow \infty} Q^{k}=\operatorname{diag}\left(\frac{1}{n} \mathbb{1}^{T}\right)(\Phi \otimes I) \\
& \lim _{k \rightarrow \infty} \tilde{Q}^{k}=\operatorname{diag}\left(\frac{1}{n^{2}} \mathbb{1} \mathbb{1}^{T}\right)(\Phi \otimes I)
\end{aligned}
$$

where the matrices $\operatorname{diag}\left(\frac{1}{n} \mathbb{1} \mathbb{1}^{T}\right)$ and $\operatorname{diag}\left(\frac{1}{n^{2}} \mathbb{1} \mathbb{1}^{T}\right)$ are block diagonal matrices of dimension $n s \times n s$ and $n^{2} s \times n^{2} s$ respectively and $\Phi$ is a stochastic matrix given by

$$
(\Phi)_{i j}= \begin{cases}\lim _{k \rightarrow \infty}\left(P^{k}\right)_{i j} & \text { if the limit exists } \\ \left(P^{l}\right)_{i j} & \text { if } P^{d}=P, l \in\{0, \ldots, d\} .\end{cases}
$$

Proof: Similar to (8) each block matrix $\left(Q^{k}\right)_{i j}$ of the matrix $Q^{k}$ can be expressed as

$$
\left(Q^{k}\right)_{i j}=\sum_{1 \leq i_{1}, \ldots i_{k-1} \leq s} p_{i i_{1}} A_{i i_{1}} p_{i_{1} i_{2}} A_{i_{1} i_{2}} \ldots p_{i_{k-1} j} A_{i_{k-1} j}
$$

where this time $A_{i j}=F_{i}, \forall j$. The reader should notice that if we sum each of the probabilities product multiplying the product of matrices in (15) we get $p_{i j}^{(k)}$ which is the $(i, j)$ entry of the matrix $P^{k}$. Each of these product of probabilities represent possible paths (sequence of transitions) of length $k$ from state $i$ to state $j$. By an inductive argument we can show that each of the $n \times n$ blocks of the matrix $Q^{k}$ can be expressed as $\left(Q^{k}\right)_{i j}=p_{i j}^{(k)} A_{i j}^{(k)}$, where $p_{i j}^{(k)}$ is the $(i j)^{t h}$ entry of the matrix $P^{k}$ and $A_{i j}^{(k)}$ are some $n \times n$ (doubly) stochastic matrices.

By the irreducibility assumption we can always find a path of state transitions which starts at a state $i$ and ends at a state $j$ which contains (at least once) every possible transition (hence transitions with nonzero probability) in the Markov chain. Let $l$ denote the length of such a path. Clearly the probability of this path is nonzero and has the general form $p_{i i_{1}} p_{i_{1} i_{2}} \ldots p_{i_{l-1} j}$, with $1 \leq i_{1}, \ldots, i_{l-1} \leq s$. Then the previously mentioned product of probabilities will be part of the conditional probability $p_{i j}^{(l)}=\left(P^{l}\right)_{i j}$. Then by (15) $p_{i i_{1}} p_{i_{1} i_{2}} \ldots p_{i_{l-1} j}$ is also encountered in the block matrix $\left(Q^{l}\right)_{i j}$ multiplying a sequence of matrices $F_{i}$, with $1 \leq i \leq s$. Because this product of probabilities contains all possible paths (and a matrix $F_{i}$ is associated to a transition from state $i$ to any other state), then each of the matrices $F_{i}$ will appear at least once. On the contrary we would have an isolated state which contradicts the hypothesis of irreducibility. We can rewrite the block matrix $\left(Q^{l}\right)_{i j}$ as follows

$$
\left(Q^{l}\right)_{i j}=p_{i i_{1}} p_{i_{1} i_{2}} \ldots p_{i_{l-1} j} F_{i} F_{i_{1}} \ldots F_{i_{l-1}}+\alpha,
$$

where we denoted by $\alpha$ the rest of the terms in the sum. By Lemma 4.2 the product of matrices $F_{i} F_{i_{1}} \ldots F_{i_{l-1}}$ is an ergodic (doubly) stochastic matrix. We mentioned above that $\left(Q^{l}\right)_{i j}$ can be expressed as $\left(Q^{l}\right)_{i j}=p_{i j}^{(l)} A_{i j}^{(l)}$, where $A_{i j}^{(l)}$ is a doubly stochastic matrix. Then $A_{i j}^{(l)}=$ $1 / p_{i j}^{(l)}\left(p_{i i_{1}} p_{i_{1} i_{2}} \ldots p_{i_{l-1} j} F_{i} F_{i_{1}} \ldots F_{i_{l-1}}+\alpha\right)$. From the ergodicity of $F_{i} F_{i_{1}} \ldots F_{i_{l-1}}$ we can deduce immediately the ergodicity of $A_{i j}^{(l)}$. Let $l^{*}$ be the largest path length such that we can arrive from any state $i$ to any state $j$ by traveling through all possible state transitions. Then from the above argument each $n \times n$ block of the matrix $Q^{l^{*}}$ is given by $\left(Q^{l^{*}}\right)_{i j}=p_{i j}^{\left(l^{*}\right)} A_{i j}^{\left(l^{*}\right)}$ where for the non-zero matrix blocks we have that $A_{i j}^{\left(l^{*}\right)}$ are ergodic (doubly) stochastic matrices. Some block can be zero if the conditional probability $p_{i j}^{\left(l^{*}\right)}$ is zero. Then since $\lim _{k \rightarrow \infty} Q^{k}=\lim _{k \rightarrow \infty}\left(Q^{l^{*}}\right)^{k}$, by Lemma 4.3 we can determine (12). In order to show (13) we follow the same line as before and use the part of Lemma 4.2 involving the Kronecker products.

At this point we are ready for the proof of Theorem 4.1.

\section{A. Sufficiency}

Proof: Note first that the stochastic system (1) represents a discrete-time Markovian jump linear system for which we can use results from the theory of Markovian jump linear systems [2] to analyze it.

By defining the error vector $e(k)$ as

$$
e(k)=X(k)-a v\left(X_{0}\right) \mathbb{1},
$$

showing the almost sure convergence of the state vector to average consensus, it is equivalent to showing that

$$
\|e(k)\|^{2} \stackrel{a . s}{\longrightarrow} 0 \text {, }
$$

where by $\|\cdot\|$ we denoted the Euclidean norm.

Without loss of generality assume that the Markov chain $\theta(k)$ is irreducible and (positive) recurrent. Thus we have only one closed set of states corresponding to a jointly connected set of graphs $\left\{G_{i}\right\}_{i=1}^{s}$. At the end of the sufficiency proof we will explain why we do not loose generality by making this assumption.

Let the $n \times n$ symmetric matrix $Q(k)$ denote the second moment of the state vector $X(k)$

$$
Q(k)=E\left[X(k) X(k)^{T}\right]
$$


where we used $E$ to denote the expectation operator. Using an approach similar to [2], consider the matrices $Q_{i}(k)$

$$
Q_{i}(k)=E\left[X(k) X(k)^{T} \chi_{\{\theta(k)=i\}}\right], i \in \mathcal{S}
$$

where $\chi_{\{\theta(k)=i\}}$ is the indicator function of the event $\{\theta(k)=i\}$. Then the second moment $Q(k)$ can be expressed as the following sum:

$$
Q(k)=\sum_{i=1}^{s} Q_{i}(k) .
$$

The set of discrete coupled Lyapunov equations governing the evolution of the matrices $Q_{i}(k)$ is given by:

$$
Q_{i}(k+1)=\sum_{j=1}^{s} p_{j i} F_{j} Q_{j}(k) F_{j}^{T}, i \in \mathcal{S},
$$

with initial conditions $Q_{i}(0)=q_{i} X_{0} X_{0}^{T}$ where $q_{i}=$ $\operatorname{Pr}(\theta(0)=i)$.

We can further obtain a vectorized form of equations (19) with the advantage of getting a discrete linear system

$$
\eta(k+1)=\Lambda \eta(k)
$$

where $\eta(k)$ is an $n^{2} s$ dimensional vector formed by the columns of all matrices $Q_{i}(k)$ and $\Lambda$ is an $n^{2} s \times n^{2} s$ matrix with each $n^{2} \times n^{2}$ block given by $(\Lambda)_{i j}=p_{j i} F_{j} \otimes F_{j}$. The initial vector $\eta(0)$ has the following structure

$$
\begin{gathered}
\eta(0)^{T}=\left[q_{1} \operatorname{col}_{1}\left(X_{0} X_{0}^{T}\right)^{T}, \ldots, q_{1} \operatorname{col}_{n}\left(X_{0} X_{0}^{T}\right)^{T}, \ldots\right. \\
\left.\ldots, q_{s} \operatorname{col}_{1}\left(X_{0} X_{0}^{T}\right)^{T}, \ldots, q_{s} \operatorname{col}_{n}\left(X_{0} X_{0}^{T}\right)^{T}\right],
\end{gathered}
$$

where by $\mathrm{col}_{i}$ we understand the $i^{t h}$ column of the considered matrix. We notice that the current setup satisfies all the conditions of Lemma 4.4 (matrix $\Lambda$ is just a transposed version of the matrix $\tilde{Q}$ in (11)) and hence we get

$$
\lim _{k \rightarrow \infty} \Lambda^{k}=\left(\Phi^{T} \otimes I\right) \operatorname{diag}\left(\frac{1}{n^{2}} \mathbb{1} \mathbb{1}^{T}\right)
$$

where $\Phi$ is given by (14). Using the observation that

$\frac{\mathbb{1}^{T}}{n^{2}}\left[q_{i} \operatorname{col}_{1}\left(X_{0} X_{0}^{T}\right)^{T}, \ldots, q_{i} \operatorname{col}_{n}\left(X_{0} X_{0}^{T}\right)^{T}\right]=\operatorname{av}\left(X_{0}\right)^{2} q_{i} \mathbb{1}^{T}$,

the limiting value of vector $\eta(k)$ is given by

$$
\lim _{k \rightarrow \infty} \eta(k)^{T}=a v\left(X_{0}\right)^{2}\left[\sum_{j=1}^{s} \Phi_{j 1} q_{j} \mathbb{1}^{T}, \ldots \sum_{j=1}^{s} \Phi_{j s} q_{j} \mathbb{1}^{T}\right],
$$

where $\Phi_{i j}$ are entries of the stochastic matrix defined in (14). By collecting the entries of $\lim _{k \rightarrow \infty} \eta(k)$ we obtain

$$
\lim _{k \rightarrow \infty} Q_{i}(k)=\operatorname{av}\left(X_{0}\right)^{2}\left(\sum_{j=1}^{s} \Phi_{j i} q_{j}\right) \mathbb{1}^{T}
$$

and from (18) we finally obtain

$$
\lim _{k \rightarrow \infty} Q(k)=a v\left(X_{0}\right)^{2} \mathbb{1}^{T}
$$

since $\sum_{i, j=1}^{s} \Phi_{j i} q_{j}=1$.

Through an almost identical process as in the case of the second moment we find that

$$
\lim _{k \rightarrow \infty} E[X(k)]=a v\left(X_{0}\right) \mathbb{1} .
$$

From (21) and (22) we deduce that the second moment of the error vector converges asymptotically (and thus exponentially)

$$
\lim _{k \rightarrow \infty} E\left[e(k) e(k)^{T}\right]=0,
$$

and therefore we can find some positive constants $\alpha$ and $\beta$ such that the following is satisfied

$$
E\left[\|e(k)\|^{2}\right]=\operatorname{trace}\left(E\left[e(k) e(k)^{T}\right]\right) \leq \alpha \beta^{k}\|e(0)\|^{2},
$$

for any positive $k$. Then by the generalized Markov inequality and by the first Borel-Cantelli Lemma [5] we conclude the almost sure convergence of the error vector to zero and implicitly the almost sure convergence of the state vector to average consensus.

We now turn to explain why it was enough to assume the Markov chain to be represented by a single closed irreducible and (positive) recurrent set of states. According to the initial distribution, with some probability the initial state will belong to either a closed positive recurrent set of states or to the transient set. The first case corresponds to the problem setup for the sufficiency proof. In the second case since the state is transient then there exist a finite positive integer $\tau$ such that $M(\tau)$ belongs to a closed set $C_{i}$. Given $X(\tau)$, we showed above that

$$
X(k) \stackrel{a . s}{\longrightarrow} a v(X(\tau)) \mathbb{1} .
$$

The state vector at time $\tau$ is given by

$$
X(\tau)=F_{i_{1}} F_{i_{2}} \ldots F_{i_{\tau-1}} X_{0}
$$

where $\left\{i_{1}, i_{2}, \ldots, i_{\tau-1}\right\}$ is a set of indices representing states in the transient set. Since the matrices $F_{i}$ are doubly stochastic we have that $\mathbb{1}^{T} F_{i}=\mathbb{1}^{T}$ for $1 \leq i \leq s$. Then

$$
a v\left(X_{\tau}\right)=\frac{\mathbb{1}^{T} X(\tau)}{n}=\frac{\mathbb{1}^{T} F_{i_{1}} \ldots F_{i_{\tau-1}} X_{0}}{n}=\operatorname{av}\left(X_{0}\right) .
$$

Therefore we have that

$$
X(k) \stackrel{a . s}{\longrightarrow} a v\left(X_{0}\right) \mathbb{1} .
$$

\section{B. Necessity}

Proof: We show that if there exist at least one irreducible closet set corresponding to a set of graphs which are not jointly connected, then there exist some initial vectors $X_{0}$ such that the state vector does not converge in probability to consensus and hence does not converge in the almost sure sense either. The complete probabilistic framework of the Markovian jump linear system (1) can be found for example in [2], pp.20. In our case the probabilistic description is rather simplified since $X_{0}$ was assumed deterministic. Let $A_{k}(\epsilon)$ define the following event $A_{k}(\epsilon)=\left\{\omega_{k}:\|e(k)\|^{2}>\epsilon\right\}$ for some positive $\epsilon$, where $e(k)$ is the error vector defined in (16).

Suppose that there exist an irreducible and positive recurrent set $C_{i^{*}}$ such that the union of the graphs in $\mathcal{G}_{i^{*}}$ are not jointly connected. Conditioning on the initial state of 
the Markov chain, the probability of the event $A_{k}(\epsilon)$ can be expressed as:

$$
\operatorname{Pr}\left(A_{k}(\epsilon)\right)=\sum_{j=1}^{s} \operatorname{Pr}\left(A_{k}(\epsilon) \mid \theta(0)=j\right) \operatorname{Pr}(\theta(0)=j) .
$$

By the assumption on the initial distribution of $\theta(k)$ we have that $\operatorname{Pr}\left(\theta(0) \in i^{*}\right)>0$. Since the union of graphs corresponding to the set $C_{i^{*}}$ does not have a spanning tree, then there exist at least two agents such that there is no paths containing these two agents. Therefore we can find an initial vector such that consensus is not reached implying that we can find an $\epsilon$ such that $\operatorname{Pr}\left(A_{k}(\epsilon) \mid \theta(0) \in i^{*}\right)$ does not converge to zero. As a consequence the state vector does not converge to consensus almost surely since it does not converge in probability to consensus.

\section{DisCUSSION AND EXTENSIONS OF THE MAIN RESULT}

We note that the proof of Theorem 3.1 is mainly based on the results of Lemma 4.2 and Lemma 4.4. The key idea of Lemma 4.4 is the fact that the matrix product $F_{i_{1}} F_{i_{2}} \ldots F_{i_{k}}$ (where $\left\{i_{1}, \ldots i_{k}\right\} \in \mathcal{S}$ is a finite set of indices which contains at least once all values between 1 and $s$ ) produces an ergodic matrix. Thus for any protocol and communication model among agents which conserve this property we can potentially show almost sure convergence to the agreement space.

We can extend our result to the case when the communications topologies are modeled by directed weighted graphs. In this case the adjacency matrix $A_{i}$ will not necessarily be symmetric and therefore the matrices $F_{i}$ will not satisfy in general the doubly stochastic property. As pointed out at the beginning of this section, in order to show the sufficiency part of Theorem 3.1 we need the matrix product $F_{i_{1}} F_{i_{2}} \ldots F_{i_{k}}$ to be ergodic. Similar to the undirected graphs case, such property is achieved if all the sets $\mathcal{G}_{i}$ are jointly strongly connected. In [10] it is shown that Lemma 4.2 holds under a weaker condition of existence of a directed spanning tree for the union of graphs in each of the sets $\mathcal{G}_{i}$. It turns out that this condition is also necessary for almost sure convergence to consensus. Thus in the case of directed graphs Theorem 3.1 can be reformulated as follows.

Theorem 5.1: Consider the stochastic system (1). Then under protocol A1 the state vector $X(k)$ converges almost surely to consensus for any initial condition $X_{0}$ if and only if each of the graphs resulting from the union of graphs in each set $\mathcal{G}_{i}$ admits a spanning tree.

As shown in [12], [13], average consensus can be reached even in the case of directed graphs if the graphs are balanced. By using protocol A1, balanced graphs generate doubly stochastic matrices $F_{i}$. Then if we assume that each of the sets $\mathcal{G}_{i}$ is either jointly strongly connected or it "jointly" posses a spanning tree, then we can show almost sure convergence to average consensus identically as in the case of undirected graphs and protocol A1. Although we will not enter in details here it turns out that having all graphs balanced is also a necessary condition to reach average consensus.

\section{CONCLUSION}

In this paper we analyzed a stochastic consensus problem for a group of agents with undirected information flow. The novelty of our approach consists in using for the first times (to the authors' knowledge) Markovian random graphs to model the communication flows among agents, which has a higher degree of generality then the ones considered until now. We showed that a necessary and sufficient condition for the state vector to converge to average consensus almost surely consists in having the sets of (undirected) graphs corresponding to the positive recurrent closed sets of the Markov chain jointly connected. Under the Markovian random graph modeling, the dynamic stochastic equation determining the evolution of the agents became a Markovian jump linear system, which proved to be instrumental in showing the almost sure convergence. Our analysis relied on several tools from algebraic matrix theory, graph theory and Markovian jump linear system theory.

\section{REFERENCES}

[1] V. Borkar and P. Varaya, "Asymptotic agreement in distributed estimation", IEEE Tr. Aut. Control, vol. AC-27, no. 3, pp. 650-655, 1982.

[2] O.L.V. Costa, M.D. Fragoso and R.P. Marques, Discrete-Time Markov Jump Linear Systems, Springer-Verlag, London 2005.

[3] J.A. Fax, "Optimal and cooperative control of vehicles formations", Ph.D. dissertation, Control Dynamical Syst., California Inst. Technol., Pasadena, CA, 2001.

[4] J.A. Fax and R.M. Murray, "Information flow and cooperative control of vehicles formations", IEEE Trans. Automatic Control, vol. 49, no. 9, pp. 1456-1476, Sept. 2004.

[5] G. Grimmet and D. Stirzaker, Probability and Random Processes, Third Edition, Oxford University Press, 2001.

[6] A. Jadbabaie, J. Lin and A.S. Morse, "Coordination of groups of mobile autonomous agents using nearest neighbor", IEEE Trans. Autom. Control, vol. 48, no. 6, pp. 998-1001, Jun 2003.

[7] Y. Hatano and M. Mesbahi, "Agreement over Random Networks", IEEE Trans. Autom. Control, vol. 50, no. 11, pp. 18671872, 2005.

[8] L. Moreau, "Stability of multi-agents systems with time-dependent communication links", IEEE Trans. Automatic Control, vol. 50, no. 2, pp. 169-182, Feb. 2005.

[9] M. Porfiri and D.J. Stilwell, "Consensus seeking over Random Directed Weighted Graphs", IEEE Tr. Aut. Control, vol. 52, no. 9, 2007.

[10] W. Ren and R.W. Beard, "Consensus seeking in multi-agents systems under dynamically changing interaction topologies", IEEE Trans. Autom. Control, vol. 50, no. 5, pp. 655-661, May 2005.

[11] R.O. Saber and R.M. Murray, "Consensus protocols for networks of dynamic agents", in Proc. 2003 Am. Control Conf., 2003, pp. 951-956.

[12] R.O. Saber and R.M. Murray, "Consensus problem in networks of agents with switching topology and time-delays", IEEE Trans. Automatic Control, vol. 49, no. 9, pp. 1520-1533, Sept. 2004.

[13] R.O. Saber, J.A. Fax and R.M. Murray, "Consensus and Cooperation in Networked Multi-Agent Systems", Proceedings of the IEEE, vol. 95, no. 1, pp. 215-233, Jan. 2007.

[14] A. Tahbaz Salehi and A. Jadbabaie, "Necessary and Sufficient Conditions for Consensus over random networks", IEEE Transactions on Automatic Control, accepted, August 2007.

[15] J.N. Tsitsiklis, "Problems in decentralized decision making and computation”, Ph.D. dissertation, Dept. Electr. Eng., Massachusetts Inst. Technol., Cambridge, MA, Nov. 1984.

[16] J.N. Tsitsiklis, D.P. Bertsekas and M. Athans, "Distributed asynchronous deterministic and stochastic gradient optimization algorithms", IEEE Trans. Aut. Control, vol. 31, no. 9, pp. 803-812, 1986.

[17] J. Wolfowitz, "Product of indecomposable aperiodic stochastic matrices", in Proc. Am. Math. Soc., 1963, vol. 15, pp. 733-736. 